UDC 004.738.5:345

Survey Paper

\title{
A Guidance Based Approach for Enhancing the e-Government Interoperability
}

\author{
Yassine Jamoussi
}

Department of Computer Science, College of Science, Sultan Qaboos University, PO Box 36, Al-Khoudh 123, Muscat, Oman

Zuhoor Al-Khanjari

Department of Computer Science, College of Science,

Sultan Qaboos University, PO Box 36, Al-Khoudh 123,

Muscat, Oman

Naoufel Kraiem

Department of Computer Science, College of Science, yessine@squ.edu.om

Sultan Qaboos University, PO Box 36, Al-Khoudh 123,

Muscat, Oman

zuhoor@squ.edu.om

naoufel@squ.edu.om

\begin{abstract}
Developing e-Government interoperability in the government context is a complex task. As interoperability in government context is associated and hindered by many challenges and barriers connected to government nature of complexity. Interoperability is generally defined as the ability for two (or more) systems to exchange information and to use the information that has been exchanged. In this paper, we focus on computing systems interoperability across government ministries to achieve interoperable e-Government IT based solutions. In order to achieve eGovernment interoperability in an organized and efficient way, this paper establishes a guidance-based approach for enhancing the e-Government Interoperability. This contribution is motivated by the limitations of the traditional software engineering methodologies in terms of analysis, design and development frameworks to a point that they can hardly cope with the growing issues of e-Government services interoperability.
\end{abstract}

Keywords: Guidance, Making a decision, Satisfaction, e-Government Services Interoperability

\section{Introduction}

E-Government interoperability is considered as significant if the interactions can take place at least at the three levels: data, services and process, with a semantics view defined in a given context $[1,2]$. However, e-Government interoperability is not an easy task to achieve. It has been recognized as a key challenge and a crucial 
issue for e-Government at least since 2001 [3, 4]. This is because, realizing eGovernment interoperability is hindered by complexity introduced in implementation. This complexity is due to the fact that initially government ministries have built their computing systems independently with specifications and solutions relevant to their particular needs but without adequate attention to the need to connect, exchange and re-use data with other systems from different ministries. This resulted in a patchwork of heterogeneous computing solutions that have limited coherence and largely are uncoordinated [5].

The rapid development of e-Government services and the growing need for integrating these electronic services has pushed forward the limitations of the software engineering methodologies in terms of analysis, design and development frameworks. Therefore, the need for more research work in developing methodological approach to solve the interoperability problem is looking ever more serious. The new approach could be coupled with an efficient implementation framework, generic enough to be used in any possible e-Government interoperability scenario.

Although some fragmented knowledge and solutions for interoperability have been accumulated since years, a practical interoperability approach is still missing. Existing approaches such as GRAI methodology, CIMOSA, PERA, etc. were developed in the context of enterprise integration rather than interoperability [6]. In the context of enterprises, interoperability refers to the ability of interactions (exchange of information and services) between enterprise systems. Besides, when addressing e-Government interoperability, one of the fundamental challenges the designers faces is to cope with process variability. Typically, for a particular process type, a multitude of process variants exists, each of them being valid in a particular context; i.e., the configuration of a particular process variant depends on concrete requirements building the process context.

Ministries are not interoperable because there are barriers to interoperability between ministries systems. Barriers are incompatibilities of various kinds at the various ministry levels. There exist common barriers to all ministries. Consequently the approach we propose aims to provide practice guidance to identify the common barriers, measure the importance of the barriers using metrics and search solutions to remove barriers [7]. Indeed, this guidance is intended to assist people who lack capacity to make their own decisions to select the appropriate strategy to ensure the interoperability between ministries systems.

In this paper we will use a combination of literature review and observation research approaches to propose a new approach to achieve seamless and practical interoperability in e-Government systems.

This paper is organized as follows. In section 2, we summarize basic concept related to interoperability. Section 3, describe the proposed guidance-based e-Government Interoperability. Section 4, presents some related work. This is followed by a discussion of the benefits of our approach in section 5 compared to the existing approaches. Finally, section 6 concludes this work with our contribution and research perspectives. 


\section{Basic Concepts Related to Interoperability}

Our proposal is based on the enhancement of the e-Government interoperability process by considering the satisfaction of different actors during this process. In particular, variability arisen during early process modeling phases is a mean of accommodating systems to changing business priorities and varying client preferences in order to satisfy all actors.

An actor satisfaction is measured by the fulfillment of his goals by the composition alternatives. In the interoperability context, actors may have antagonist interests and may not be satisfied with the same aspects. Negotiation is then adopted to solve undesired conflicts.

These concepts are integrated into an approach through which the gap between variability and satisfaction is bridged. We first introduce these concepts in more detail, before presenting our proposed approach.

\subsection{Interoperability issues}

When using the existing BPM formalisms to describe the interoperability, usually several variants are generally defined, as shown in Fig. 1. This solution usually yields to many variation points. In addition, variants are not closely related to each other because their process models are loosely coupled. In particular, the interoperability described by these points of variation does not provide support for combining or merging existing variants to new [8]. Indeed, most laws governing local bodies, such as municipalities, are defined by the central government and have an impact on the business processes and information systems of the local entities. If the laws can be formalized as formal process with variability taking into account the business and technical differences of the various municipalities, one can have great advantages from the reuse of these variants.

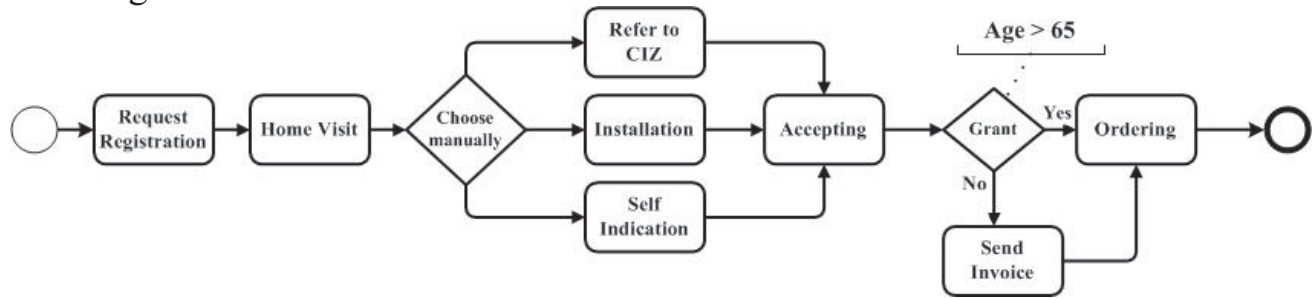

Figure 1. Process variants realized by means of conditional branches

As consequence, the interoperability of the services leads to flaws over time as the consideration of actors needs is ignored. Variants, therefore, are neither transparent nor explicitly defined. Consequently, the underlying system is unaware of the process variants and thus cannot provide sophisticated support for them; e.g., creating a view of the process model representing a particular process variant is not enabled unless the information about which process element (e.g., activity) is part of which particular process variant is explicitly included within the business process models [9]. 
In summary, neither the use of separate process models for capturing the different variants nor the definition of these variants within one model (using conditional branches) constitutes a viable solution in many cases.

\subsection{Variability}

Variability is defined as "the capacity of a system or an artifact to be changed, custom or configured in a particular use context" [10]. Variability can concern functional or non-functional aspects. In web service context, functional variability deals with the capabilities offered by the service whereas non-functional aspects concern different service quality dimensions.

Currently, in the e-Government interoperability literature, the need of accommodating a business process relays on business rules and late modeling techniques for changing BPs. However, these approaches are usually quite low-level and the possible configurations are not explicitly evaluated with respect to business goals and priorities [11].

These limits arisen the need of modeling and eliciting variability in intentional terms. Many goal-oriented formalisms are used to explore and analyze the variability.

For instance, the I* framework [12] provides a goal- and agent-oriented approach for exploring possible alternatives for creating new or improving existing business or information system situations.

Roland et al. propose a formalism that allows representing a process model, expressed in intentional terms, called MAP [13]. This formalism provides a representation mechanism based on a non-deterministic ordering of goals to be accomplished (intentions) and the different alternative ways for achieving them (strategies).

Applying those models in the web service field attracted many researchers who proposed different approaches such as exploring web services from a business value perspective, aligning web services with business strategy and different other approaches related to web service modeling, discovery, selection and composition [14].

In a previous published paper [14], we presented a new concept of service called intentional service that abstracts technical description of a service and stresses on the goal that the service can achieve. An intentional service model (IMS) for describing intentional services is proposed within this work. The IMS model is deduced from the process model described with MAP and presents a high variability composite service. Hence, in this paper we will not focus on how to define the variability model from the requirements.

\subsection{Satisfaction}

To deal with actor's satisfaction in e-Government interoperability context, four facets must be addressed: the actors, the satisfaction type, its time and functions [15]. 
Although e-Government interoperability introduced different actors and roles, almost existing approaches still address the interoperability service from unilateral perspective [16]. These strategies are independent from different providers: single service providers (SSPs) providing single services, joint venture (JV) providing composite services and strategic alliance (SA) providing both services. Providing composite service for ensuring the interoperability is a promising strategy and more interest should be accorded. Obviously, current approaches address only the enduser satisfaction by adapting interoperability process to the end-user context, the used canal or the end-user requirements [17].

In this sense, satisfaction functions are simply constraints, utility functions or end-user preferences that are considered for ensuring e-Government interoperability. In the Requirement Engineering works, satisfaction is related to goal satisfaction. This research area is concerned with the identification of the goals to be achieved by the system "to-be", the operationalization of such goals into specifications of services and constraints and the assignment of responsibilities for such services and constraints among human, physical and software components forming the system agents [18].

In GORE, the goals are used in different levels of abstraction, from the strategic goals of high-level technical goals to low-level [18]. According to [19] there are two taxonomies of goals. The first taxonomy distinguishes between functional and nonfunctional goals while the second divides goals into "hard" and "soft".

Functional goals are used for specifying what the service is expected to deliver whereas non-functional goals refer to quality requirements that the software needs to satisfy while delivering the services. Hard goals are goals which satisfaction can be established using (formal) verification techniques. However, soft goals cannot be satisfied in a clear-cut sense but only satisficed when thresholds of some precise criteria are reached [20].

\section{The proposed Approach}

In this section, the proposed approach that helps achieving interoperability between heterogeneous independent ministries' systems is presented and described.

\subsection{Approach overview}

A considerable number of research efforts on interoperability are conducted both in industry and academia. The interoperability process is similar to the process used while composing services in Service Based Application (SBA) [21]. Many initiatives for modeling SBAs have been proposed, namely languages (e.g., BPEL4WS, OWL-S, Petri nets, etc.) [22]. All these initiatives adopt a 'functiondriven' service modeling focusing on 'low level' technical statements (e.g., coordination messages, input/output parameters and bindings) that are understandable by software programmers but far to be comprehensible by end-users. However, end-users need to interact with service providers to obtain SBAs satisfying their requirements. Thus, SBAs must be modeled in terms of business 
goals and not in terms of technical statements. Hence, we adopt a 'requirementdriven' approach that allows a 'high level' modeling of SBAs [14].

Before identifying the proposed approach, which is considered as "to-be system", it is important to define the "as-is system" i.e. the current status of the existing e-Government systems [39].

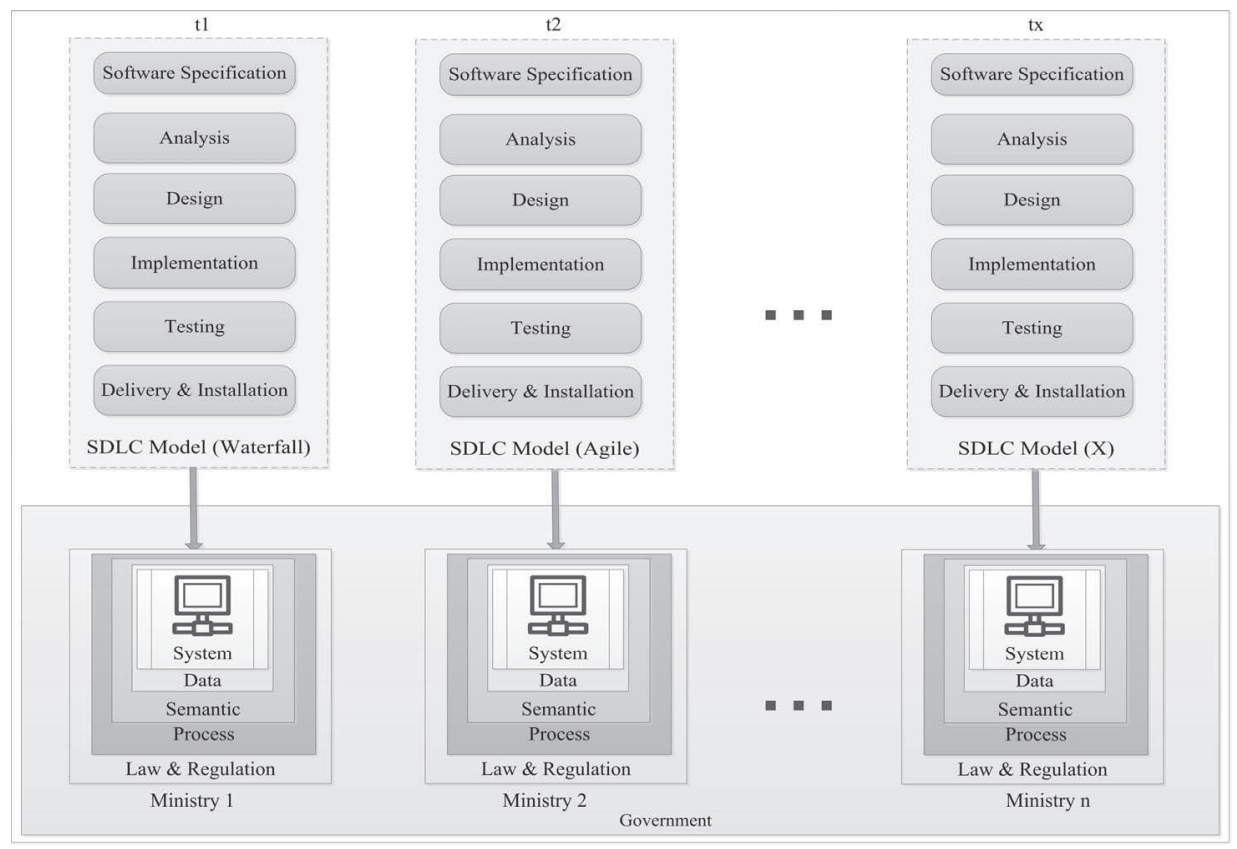

Figure 2. As-is Government Systems [39]

\subsubsection{As-Is System}

The existing status results from independent initiatives of government ministries to build their own computer systems with specifications and solutions relevant to their particular needs. Figure 2, which has been introduced by Al-Khanjari et al. [39], depicts these initiatives scenario.

These investments of utilizing computer systems in government sector result in: isolated, independent, heterogeneous computer systems that have limited coherence and largely are uncoordinated.

\subsubsection{To-Be System}

A key determinant of success in e-Government initiative is based on the ability of these isolated, independent, heterogeneous computing systems to cooperate and work together sharing information and integrating processes across all boundaries surrounding their isolated computing systems. This requires a holistic view of the "to-be system" that would combine and organize these existing systems under one umbrella. 
In this section, the proposed "to-be system" takes into consideration the existing independent software systems deployments and technical implementation rather than trying to replace them. Ministries have already invested heavily in building their running systems. It would be impractical to suggest a big bang approach that induces fundamental changes on existing infrastructures.

To enhance the e-Government interoperability, we propose an approach that is based on a multi-level framework inspired from [37, 38] (Figure 3). This approach aims to improve the e-Government interoperability by increasing the satisfaction level of requesters. Hence, through an alignment process, e-Government providers will guide the requesters to achieve successfully their tasks.

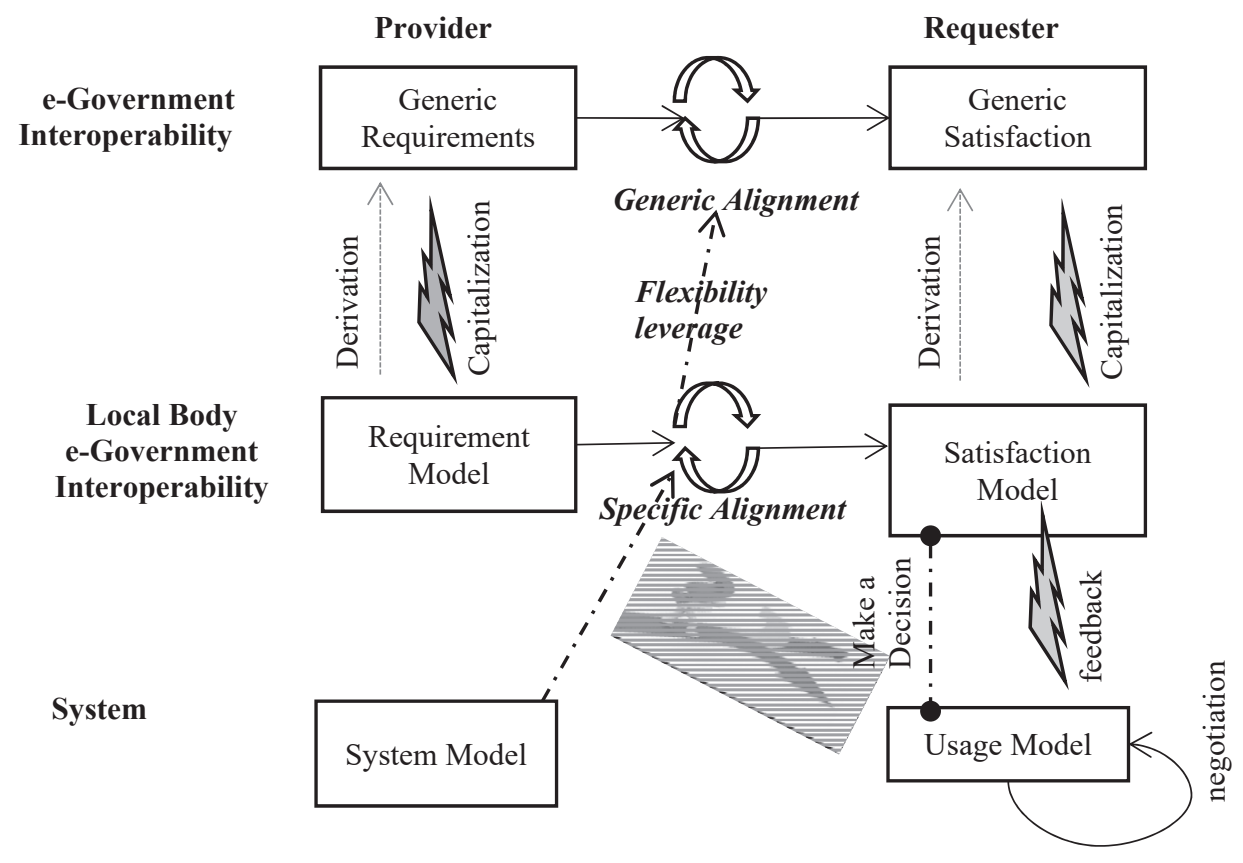

Figure 3. The proposed e-Government interoperability approach

In this approach, the e-Government is modeled according to three levels:

- e-Government level: related to the common e-Government interoperability requirements.

- Local Body level: allowing the extension of these generic interoperability e-Government interoperability requirements to the local body specific needs.

- System level: representing the e-Government system functionalities.

The suggested approach combines 2 types of alignment. Firstly, the "Generic Alignment" is achieved between the Generic Requirements (according to the provider point of view) and the Generic Satisfaction (according to the requester point of view). Secondly, the "Specific Alignment" -that is based on the generic eGovernment knowledge- is done to elaborate the target Model (Satisfaction Model). 
This approach is established in order to take into account the interoperability knowledge aspect. In this respect, the suggested approach would initially consist in the alignment of the interoperability e-Government requirements (Generic Model) and the expectations of the requesters. On this e-Government level, the process is to align the e-Government system to requester needs. A number of alignment information are collected and form flexibility leverage knowledge for the interoperability in the Specific level.

The suggested approach includes three main phases:

- Elaborate the Requirement Model RM (Modeling e-Government interoperability in business term)

- Align these specific requirements with the needs of requesters by taking into account the knowledge acquired in the generic satisfaction (Modeling Satisfaction),

- Guide the process and capitalize the interoperability knowledge.

Therefore, the first step to build the Requirements Model from the Generic Model GM is to identify the differences and similarities between these two models. Based on these differences and similarities, the Requirements Model RM can be built by derivation. In order to emphasize the variability, we have chosen to use the MAP formalism [13]. According to this formalism, the requirement models will be defined as a set of sections. Indeed, a section is the key element of a MAP. It is a triplet $<\mathrm{G}_{i}$, $\mathrm{G}_{\mathrm{j}}, \mathrm{S}_{\mathrm{ij}}>$ and represents a way to achieve the target goal $\mathrm{G}_{\mathrm{j}}$ from the source goal $\mathrm{G}_{\mathrm{i}}$

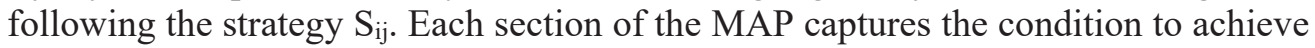
a goal and the specific manner in which the process associated with the target goal can be performed.

Then, based on the reuse of the Generic Satisfaction (done in the e-Government level), this RM will be aligned with the system to obtain the Satisfaction Model SM.

This specific alignment is implemented according to the following stages:

- For sections of the Requirements Model (RM) that have been built directly from the Generic Model, the corresponding sections in the predefined Generic Satisfaction Model are directly included into the Satisfaction Model (SM).

- For the RM sections that have slight differences (such terminologies discordance) with the Generic Model sections, the corresponding sections in the Generic Satisfaction Model are considered with reflecting the necessary modifications (based on the changes that have been made to build these specific RM sections from the Generic Model GM). Then, these sections are included in the SM.

- For RM sections with weak or non-existent similarities with the GM sections:

- If this section has been created using an approach guided by the existing so there is no need to make an alignment and this section will be therefore included, as it is, in the RM in terms of benefit. 
- Otherwise, an alignment (similar to the alignment in the Generic level) is done between this specific need and sections of the Satisfaction Model (SM) that would allow meeting this need. This newly section is then included in the RM. An attempt is made to enrich the Generic Model and Generic Satisfaction by these added sections respectively in the RM and the SM.

As stated above, the alignment process at the specific level, unlike at the generic level, is based on the analysis of the variations between "e-Government Requirements" (Generic Model) and "Specific Requirements" (Requirements Model). It should be recalled, in this regard, that few differences exist between these two levels (e-Government and Specific levels) that contribute to facilitate the analysis and make it much easier.

More details for the description and the application of the suggested approach will be presented in the next sections.

\subsection{Modeling e-Government Interoperability in business term}

We have conducted several case studies in different government departments to elaborate key requirements for the definition, adaptation, and management of interoperability process variants. This strong linkage to practice is required to realize a complete and solid approach for process variant management. The requirements we have identified are related to different aspects including the modeling of process variants, their linkage to process context, their execution in a process management system [23]. Hence we adopted in our approach a flexible interoperability process and an interoperability measurement method, which acts as a flexibility leverage.

1. Interoperability process. Although the e-Government services are constantly increasing, the complexity of their interoperability is rapidly growing. This highlights the need for a formal approach and design standards to ensure efficient and more importantly repeatable interoperability enabled services.

There is no federated systematic approach for all ministries to comply with. To help solving this challenge, this paper proposes an end-to-end framework to achieve interoperability in e-Government via methodological approach. Our vision is that all public ministries will need to share services of their respective domain in order to seamlessly exchange data and workflows. However, services sharing will not be possible if these services are not designed and implemented in a way that considers interoperability as a part of the services development process. Using service logic to only solve a single problem in a single ministry is not useful and does not leverage the logic's reuse and interoperable potential.

To help achieving e-Government interoperability between heterogeneous, independent ministries' computing systems, the interoperability process is conducted through a structured approach that aims at defining the main phases to follow in a sequential way with possible iterations between the stages [36]. Depending on whether the process is being applied to an individual ministry or a pair collaboration 
ministries [24]. This process is influenced and driven by the advantages of the other software engineering models/approaches such as Waterfall, Agile and Service oriented development methodology by Thomas Erl [25].

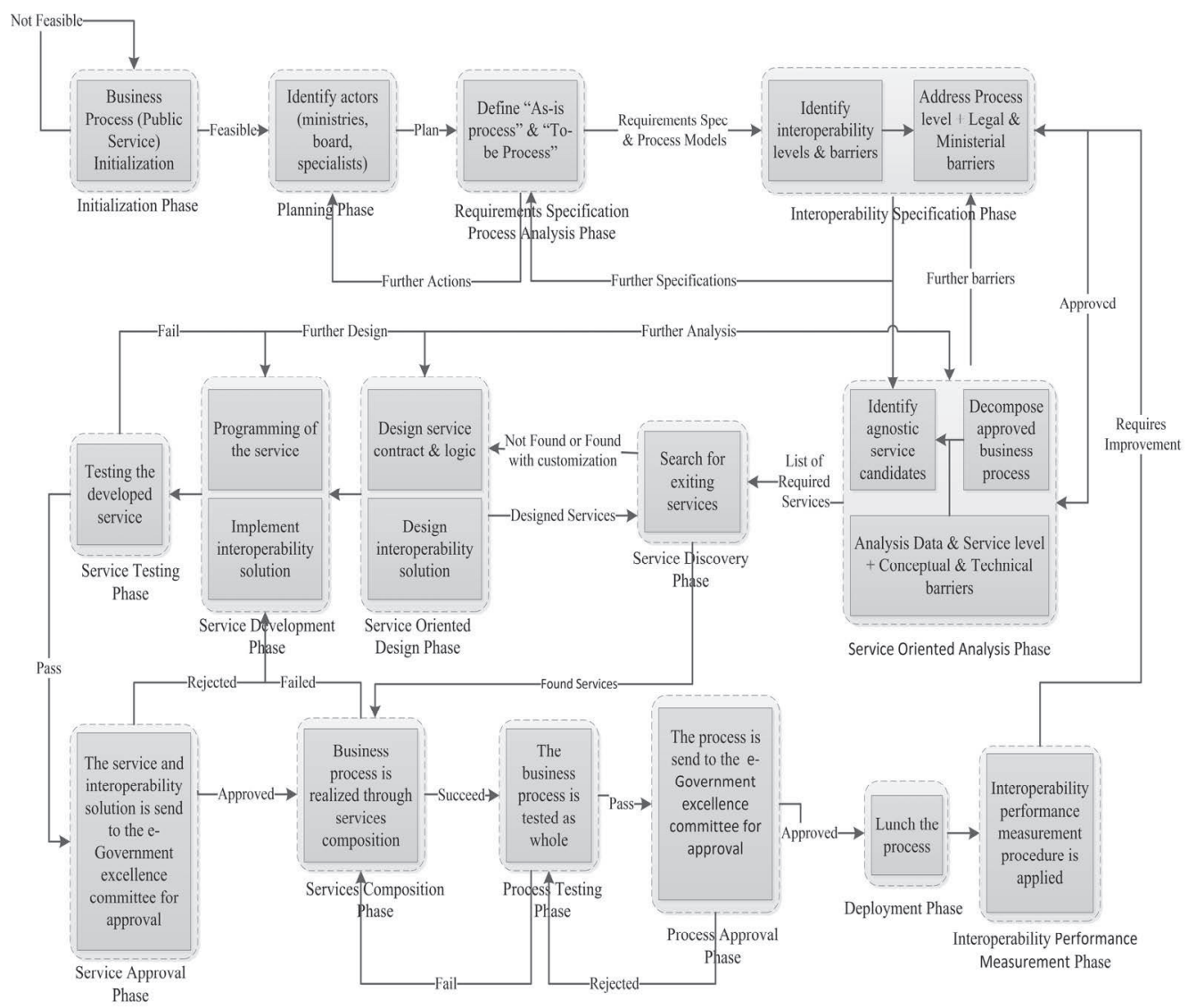

Figure 4. e-Government Interoperability process (Detailed Level) [36]

Figure 4 illustrates all stages described above of the e-Government Interoperability Driven process in a detailed level showing all possible iterations between phases. Practically the activities of these phases can be integrated with the activities of the previous phases. The need for interoperability at each level of concern (Process, Service, and Data) associated with the "to-be" automated business process is identified. Then, the associated barriers with each level to interoperability are identified. Besides, during the composition stage, the "to-be" automated business process is realized through the services composition. These services are approved by the e-Government Excellence Committee make it possible to achieve ministry to ministry interoperability from interconnection services offered by multiple ministry partners based on business process. 
2. Flexibility leverage. The Daclin's interoperability compatibility measurement method will be used during this stage [7]. This measure is performed when the partner (ministries)/system of the interoperation is known. The measure is done with respect to the identified barriers to interoperability. Referring to each interoperability concern (level) and interoperability barrier, the objective is to check if there is incompatibility or not. With regards to the interoperability barriers, if an incompatibility is detected, the coefficient 1 is assigned to the interoperating level and the barrier that are considered. Conversely, the coefficient 0 will be assigned when none incompatibility is detected. Following this rule, the compatibility measurement matrix proposed by Al-Khanjari et al. [36] is used as presented in Table 1 to represent the interoperability levels that must be achieved between concerned ministries and the barriers that must be removed to achieve each interoperability level.

To reach highest degree of compatibility means that all the barriers to interoperability have been removed. The opposite situation means the poorest degree of interoperability. The compatibility measure allows ministries to know what kinds of barriers there are and what barriers have to be removed so that interoperability can be improved. In a similar way, the incompatibility measurement can allow ministry to prioritize the actions to be taken to improve interoperability. It is also necessary to work with interoperation ministries so that concerted and common actions to remove these barriers are taken at both sides.

\begin{tabular}{|l|c|c|c|c|c|}
\hline \multirow{2}{*}{$\begin{array}{c}\text { Barriers } \\
\text { Levels }\end{array}$} & \multicolumn{2}{|c|}{ Conceptual } & Ministerial & Legal & Technical \\
\cline { 2 - 6 } & Syntactic & Semantic & & & \\
\hline Process & 1 & 0 & 1 & 1 & 1 \\
\hline Service & 0 & 1 & 0 & 0 & 1 \\
\hline Data & 1 & 1 & 1 & 1 & 1 \\
\hline
\end{tabular}

Table 1. The Compatibility Measurement Matrix [36]

After the compatibility measurement matrix is filled, the legal barrier is removed (if exist) by the project board members and they escalate it to other parties if required. As it is always not possible to proceed with project unless it is aligned with the country's laws and regulations.

Meanwhile, the ministry barrier is removed as well by removing any process conflicts and authorities/responsibilities clearly defined between involved ministries. If for any reason the process activities not agreed upon or legal or ministerial barriers are not aligned with the proposed "to-be" business process, then, the proposed "to-be" business process is sent back to the previous phase for further analysis or adjustment. Otherwise, if all involved parties approve the process act activities and the legal and ministerial barriers are removed then the "to-be" business process is sent to the project board for approval and moved to the next phase. 


\subsection{Modeling Satisfaction}

Our approach supports actors' satisfaction all over the interoperability process. Besides, the expectations defined at the service-level agreement (SLA) [38], we elicit actors' satisfaction at an early phase through soft goals that are imprecise, subjective, idealistic and context-specific goals [19]. Taking into account an actor satisfaction all over the interoperability process is motivated by the influence of past and current experience on the future perceptions. This idea is clearly expressed in the marketing research area that distinguishes between transaction-specific satisfaction and cumulative satisfaction [26]. While transaction-specific satisfaction may provide specific diagnostic information about a particular service encounter, cumulative satisfaction is concerned with all of consumer's previous experiences with a firm, product, or service cumulatively [26].

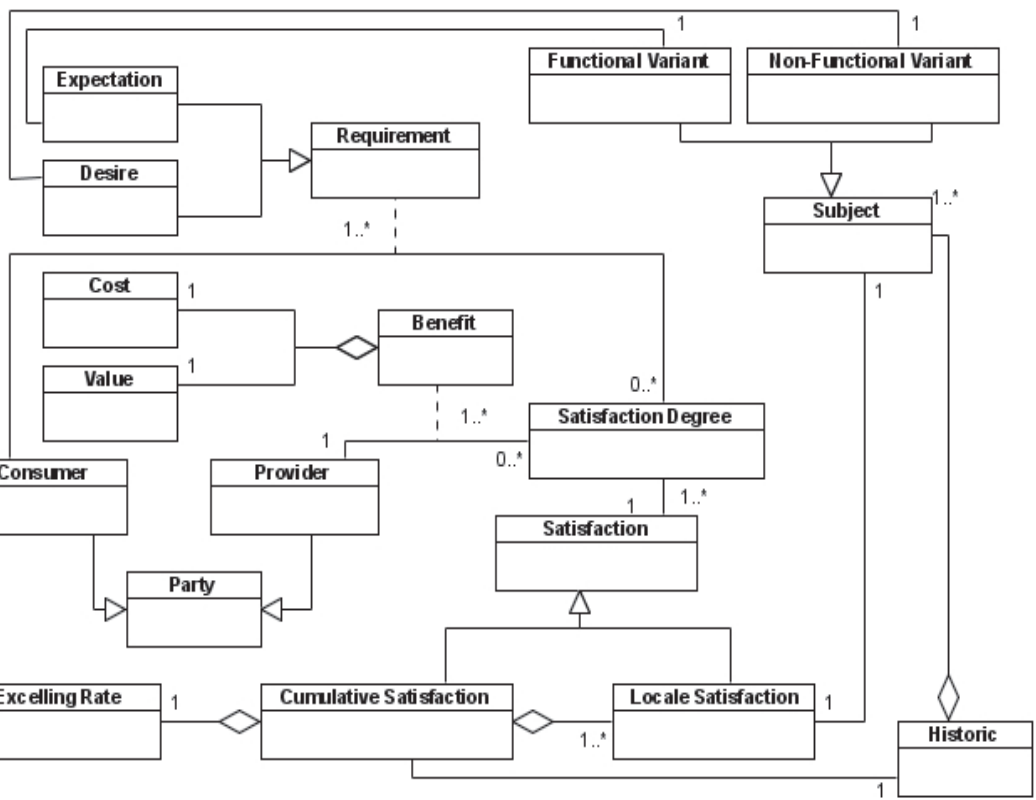

Figure 5. Generic Satisfaction (meta-model)

Taking the state-based conceptualization of satisfaction, we define a local satisfaction measurement relative to each step of the interoperability process and a cumulative one relative to all the achieved steps in the process. To measure the cumulative satisfaction, we introduce the excelling concept suggested by [19]. In [19], authors noticed that satisfying soft goals does not cover situations in which continual improvement of thresholds is expected. They introduced the excelling notion to express this need. We also introduce the concept of the satisfaction degree as a customized measure of the satisfaction. The satisfaction degree plays the role of the negotiation decision function. All these concepts are presented in the proposed meta-model of Figure 5. 
Although, these concepts are proposed in the consumer satisfaction context, we believe that they also reflect the buyer satisfaction. The difference between the two parties resides on the satisfaction factors. We introduce for each actor his satisfaction factors and a method to measure them.

1. Government Satisfaction. As mentioned, the alignment and therefore the government satisfaction are translated in terms of benefits [27]. Benefit is the difference between the value and the utility of a service. According to our approach, the government satisfaction should be considered at different steps. For this end, we need to evaluate the benefit of functional choices (interoperability strategies) and non-functional choices (concrete services among a service community). To measure an interoperability strategy benefit, we use the following technique:

A decision model for the departmental government is built. This model define an hierarchy of soft goals that reflects the long-term vision. The top of this hierarchy is the main goal and the lower levels are the refinement of the main goal in more concrete goals. For example, as shown in Figure 6, the mission of a departmental government is to become the first virtual provider of an e-Government service.

We enrich the goal model by numerical annotations showing the contribution of each subgoal to goals of the upper level. To rank these contributions, we use the smart method and give ' 1 ' to the weakest contribution and multiply it to more important contributions. For instance, satisfying its consumers has a high contribution to the e-Government mission.

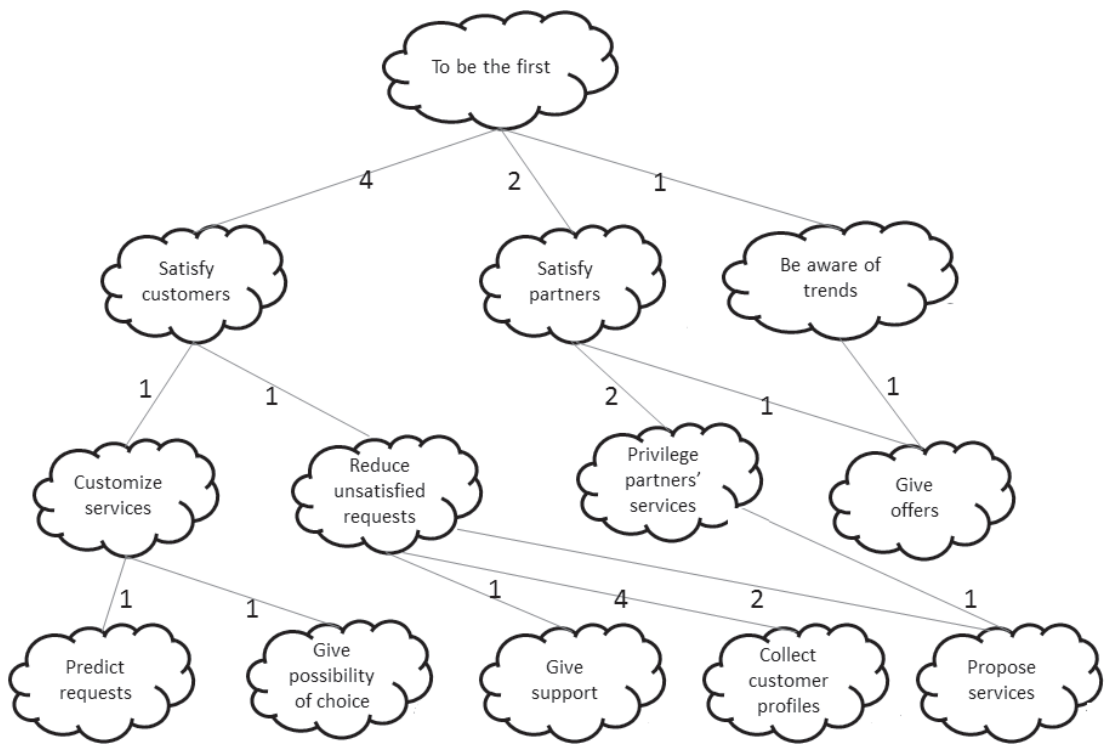

Figure 6. Provider decision model

The contribution of each leaf goal to the mission is calculated by an inference rule defined as follow: If $\mathrm{C}\left(\mathrm{O}_{\mathrm{i}} / \mathrm{O}_{\mathrm{j}}\right)=\mathrm{x}$ and $\mathrm{C}\left(\mathrm{O}_{\mathrm{j}} / \mathrm{O}_{\mathrm{k}}\right)=\mathrm{y}$ then $\mathrm{C}\left(\mathrm{O}_{\mathrm{i}} / \mathrm{O}_{\mathrm{k}}\right)=\mathrm{x}^{*} \mathrm{y}, \mathrm{O}_{\mathrm{i}}, \mathrm{O}_{\mathrm{j}}$ and $\mathrm{O}_{\mathrm{k}}$ are goals. 
To measure the contribution of a service variant to the enterprise mission, we use a method similar to that exposed on [27]. This approach uses a goal-oriented requirement engineering approach to evaluate the contribution of each section to the leaf goals of the goal model. This contribution is first estimated in terms of value then in terms of utility. The contribution of each section to the organization mission is then calculated.

Finally, we use the following rules to calculate the benefit and the value of each service:

- The benefit of an e-Government service directly deduced from its atomic services.

- At a variation point, the maximum of the variant values is selected to evaluate the aggregate service.

- At a composition point, the component values are summed.

Unlike functional strategy evaluation, which is done in a static manner, measuring the benefit of a concrete service is done dynamically. The value and benefit of a service are determined according to its non-functional attributes. In our current work, we consider only utility and response time. In [17], author mentions the importance of both services to appreciate the integrated web service.

The added value of a web service is defined by $t^{*} \mathrm{x}$ where $\mathrm{t}$ is the utility of the service and $\mathrm{x}$ is an average of the number of service used per year by one departmental government. In the case where the service requester is in turn another government provider he will inflict an added $\mathrm{t}^{\prime}{ }^{*} \mathrm{y}$ benefit on his consumer where, $\mathrm{t}$ ' is the utility of the integrated service and $\mathrm{y}$ is the number of requested atomic services composing the service in question. To calculate the utility of the integrated service, the Government Provider (GP) should take into consideration that consumers expect that getting an integrated service is harder than getting all its components separately. Thus, the more the composite service gathers few atomic services provided by different departments, the more the GP is lucky to win benefit. To measure the provider satisfaction after executing each service, the real benefit is calculated.

Finally, to bind benefit with the satisfaction degree, the provider can clearly make assumptions such as "A benefit over 10 unities satisfies me to $80 \%$ ".

2. Requester satisfaction. In the context of e-Government interoperability, satisfaction factors encompass service attributes, system attributes and information quality attributes [28]. Although requirement engineering has studied extensively end-user satisfaction, it doesn't focus on the process of satisfaction formation [28]. In the marketing literature, on the other hand, many models are provided to describe satisfaction formation. An important framework for understanding the satisfaction formation process is the disconfirmation paradigm. According to this theory, satisfaction is determined by the discrepancy between perceived performance and cognitive standards such as expectations and desires [29]. On line with those paradigms, we propose a model of user satisfaction based on his expectations and desires, which distinguishes between two stages: before the execution time and after 
it. To determine the user satisfaction at the discovery and selection times, we use a technique similar to the government provider case. However, unlike government provider, the service requester has a short-term vision of its goals and objectives. In this sense a decision model is associated to each discovery and selection step. For instance, a government search service satisfies the user if he makes a minimum effort and gives him pertinent results. However a good government service is a service that allows him to customize his choices. To select a concrete service, the user may require a high security rate for a payment service and not wonder about the security rate of the government service. The decision model is also a goal model described with soft goals related to the current step.

After the service execution step, the user is asked to give a macro feedback of his impressions about the adopted service on the basis of perceived performance. He is asked to answer the following questions: "To what extent the service fulfills your expectations?" and "To what extent the service fulfills your desires?" User's responses to these questions are mainly qualitative and subjective. In such kinds of situations, fuzzy values are adequate. As proposed in [30], response can be possibly performed by linguistic variables like: "bad", "poor", "fair", "good" and "excellent". A triangular fuzzy number is associated to each linguistic value. Finally, assuming that expectations and desires have the same weight for the user, the two assessments are summed and then defuzzified with the center of area method to get a non- fuzzy value expressing the user satisfaction degree.

\subsection{Guidance-based process to support the Interoperability}

The main aim of the proposed approach is to guide user during the interoperability process. Hence we adopted a meta-strategy. The motivation of the meta-strategy is to guide a negotiator to make his negotiation decision on the basis of a judgment on a negotiation situation, which takes into account the actual satisfaction degree, the cumulative satisfaction value, the opponent behavior and anything he finds important to make his decision. For instance, a negotiation situation can be qualified as bad if the satisfaction degree is in a continuous decrease; he is conceding more than his opponent or the later does not respect his commitments.

Based on the literature review [31,32] we ended up with the meta-strategy illustrated in Figure 7. This latter describes the meta-strategy using the MAP formalism [13]. Indeed, this latter allows specifying process models in a flexible way by focusing on the process intentions and on the various ways to achieve them. Making a decision in any negotiation situation may lead to one of the three following states: the negotiator may maintain the same satisfaction degree, decrease, or increase it. These three states represent negotiator intentions. To reach an intention in the MAP model, strategies are used. Three types of strategies are proposed in the negotiation literature:

- Concession strategy allows negotiator to decrease, within the acceptance range, his utility function to reach an agreement. Many tactics can be used to generate concession. For instance, time dependent tactics are adapted if the time has an impact in the negotiator decision, behavior dependent are used 
when a negotiator tries to imitate the opponent's behavior and resource dependent can be applied if the negotiator takes into account the existing resources.

- Trade-off strategy allows a negotiator to make offers that keep his same satisfaction degree as in the previous step, but expecting to be more acceptable for its opponent. A trade-off algorithm is proposed by [33]. The idea of this algorithm is to generate propositions that increase progressively some variables values.

- Argumentation strategy allows negotiators to add explications or to exercise persuasion forces on opponents. Possible tactics for persuasion are threats, rewards, appeals and explications. By using different arguments, a negotiator can increase or maintain his satisfaction degree by convincing his opponent to accept his offer.

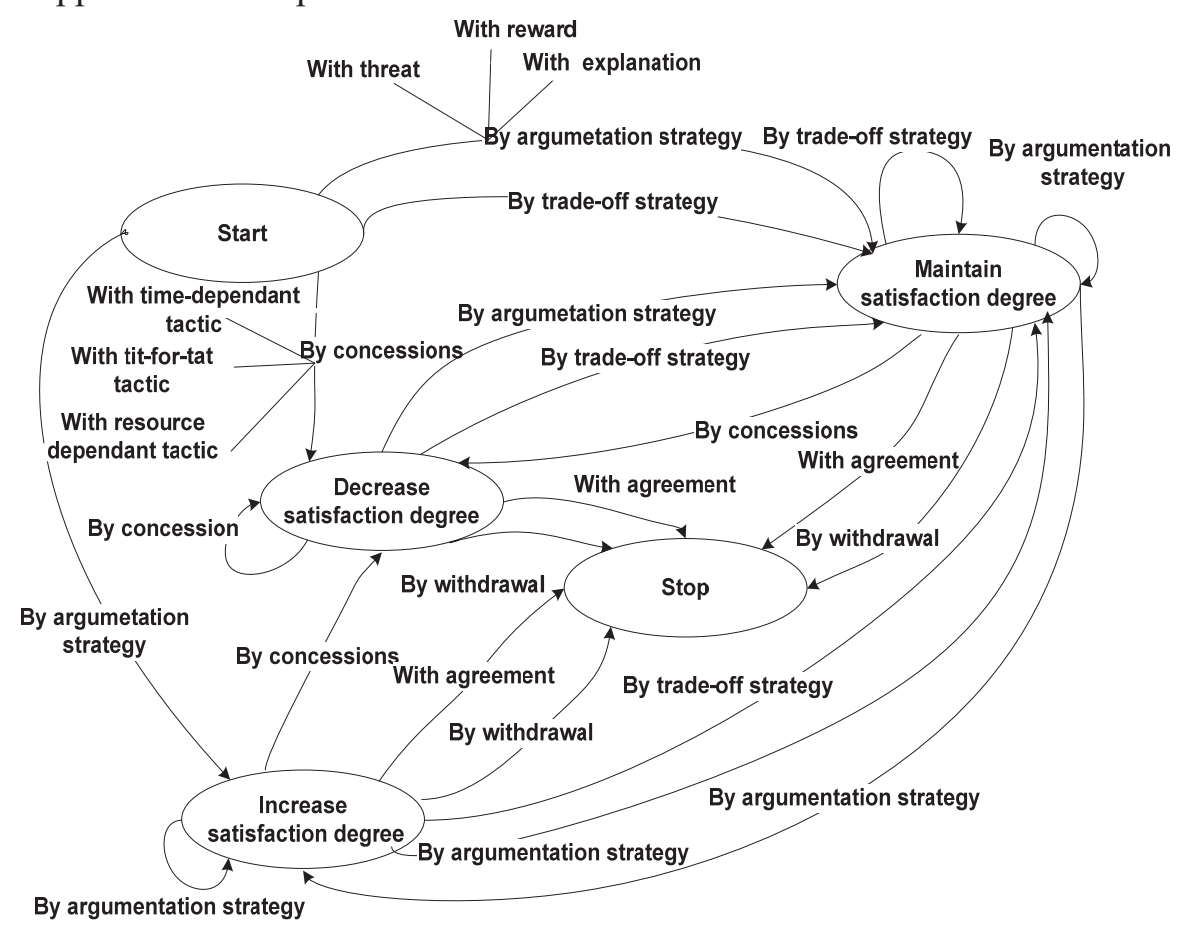

Figure 7. Negotiation meta-strategy

\section{Related Work}

There are always alternatives when working on information systems generally and interoperability in particular. Therefore, some individual researchers tried to tackle the interoperability challenge by developing some methodologies that provides a guide on how to implement interoperability solutions between enterprises (ministries) systems. This approach aims at defining the main steps to follow in a sequential way. 
In 2005, Daclin proposed a methodology following federated approach [7]. This methodology establishes interoperability at the business level only. Moreover, it is very generic and lacks detailed steps to be followed by interoperability solution implementer. However, it can help in drawing guidelines for detailed methodologies.

In 2007, Sanati and colleagues proposed a new methodology called "E-Service Integration Methodology" by which the interoperability is considered as part of software development phases [33]. This methodology doesn't address interoperability barriers in details. Moreover, it lacks the implementation details which requires further research that focus on detailing the integration specific tasks of E-SIM to clarify such tasks in their depth.

In 2008, Daclin and colleagues proposed another methodology for enterprise interoperability. It aims "to provide a generic methodology allowing enterprises identifying their problems in terms of interoperability and selecting solutions adapted to their needs" [34].

This methodology is more detailed than Daclin's methodology and provided the compatibility measurement matrix (Table 1) to identify the barriers over all concerns before implementing the interoperability solution. However, this methodology was considered interoperability only between two partners [7]. Therefore, it is not applicable to be used for e-Government solution where the interoperability must be considered between many partners sometimes.

In 2009, Saekow and Boonmee proposed a Pragmatic Approach to Interoperability Practical Implementation Support (IPIS) [28] to approach eGovernment interoperability. They described an overall methodology for IPIS approach in order to fully engage the e-Government interoperability.

In 2014, Al-Khanjari et al. proposed a new methodology called "E-Service Integration Methodology" by which the interoperability is considered as part of software development phases [36]. This methodology doesn't address guidance. Moreover, it lacks the implementation details, which requires further research that focus on detailing the integration specific tasks of E-SIM to clarify such tasks in their depth.

Even though this methodology is detailed enough in terms of combining existing solutions, this methodology is dedicated with IPIS tools with a main purpose to help adapting standards to only achieve technical interoperability. Still, this methodology does not address the other levels and barriers of the Interoperability.

\section{Discussion and Conclusion}

As can be seen, the suggested approach can be used to satisfy a number of limitations and problems of the traditional software engineering methodologies in terms of analysis, design and development frameworks to a point that they can hardly cope with the growing issues of e-Government services interoperability. As well, investments of utilizing computer systems in government sector result in: isolated, independent, heterogeneous computer systems that have limited coherence and largely are uncoordinated. 
The development of this approach is taking into consideration the following points:

- First: it considers the existing independent software systems deployments and technical implementation rather than trying to replace them. Ministries have already invested heavily in building their running systems and it would be impractical to suggest a big bang approach that induces fundamental changes on existing infrastructures.

- Second: it is interoperability focused. So it considers the interoperability identification and elimination as one of the main phases in the proposed approach.

- Third: this approach is built upon three levels (Generic, local body, system) and two perspectives (provider and the requester). The three levels give a complete picture about the know how required to system to achieve eGovernment Interoperability. The two perspectives provides enough anchor points for systems to coordinate their activity without fully pre-specifying the capabilities and specifications of the e-Governments and therefore enhancing the flexibility of e-Government system.

Hence, using the proposed approach will avoid hazardous interoperability problems and therefore, reduce the time needed to develop interoperability and avoid the implementation of non-adapted solutions. Here are some benefits of the proposed approach.

- This approach will be generic enough to be used in any e-Government public service.

- It allows ministries to identify their problems in terms of interoperability and select solutions adapted to their needs.

- Unified approach to e-Government projects allows following a structured approach in a step-by-step manner in order to guide ministries during the interoperability implementation of solutions.

- Evaluating interoperability degree between ministries to know their strengths and weaknesses.

- Dynamically composing available interoperability solution services according to identified requirements.

- Focused on identifying and involving various actors and stakeholders of the ministries concerned.

We compared our proposed approach with other approaches discussed above in the related work. The result of this comparison study highlights the contribution value of the proposed approach and architecture in the body of knowledge. This study's contribution linked between many concepts in software engineering discipline. Besides, it linked between Service Oriented Architecture (SOA), Service Oriented Development Life Cycle (SODLC), e-Government and Interoperability. 
The work presented in this paper opens the way to different perspectives and can be continued in several directions. These perspectives can be related to two levels: that of continuity of the work done and the enlargement of the research area.

- Conduct an empirical study: The empirical study lead as a series of controlled experiments allows validating the ability of the suggested approach to solve the existing problems. Nevertheless, we believe that further experiments should be conducted to confirm the validation of the various research hypotheses formulated through this paper. We propose, to this end, to extend these experiences to other samples of subjects and/or other local body e-Government departments. This would allow us to deduce improvements.

- Automate the approach: The large number of manipulated data (for big structures) makes very difficult the manual application of the alignment process. So the automation of the approach seems essential for using it in large projects and implemented by some ministries or administrations. A software environment would be a logical continuation of this work.

\section{References}

[1] IDEAS (2003), IDEAS Project Deliverables (WP1-WP7), Public reports [online]. Available: www.ideas-road map.net.

[2] IEEE. (2012). Standards Glossary. [online]. Available:http://www.ieee.org/education_careers/education/standards/standa rds_glossary.html.

[3] Moen, W. E. (2001). The metadata approach to accessing government information. Government Information Quarterly, 18(3), 155-165.

[4] Gatautis, R., Kulvietis, G., \& Vitkauskaite, E. (2015). Lithuanian eGovernment interoperability model. Engineering Economics, 62(2).

[5] United Nations Development Program. (UNDP) (2007). e-Government interoperability: Guide. Bangkok, Thailand: United Nations Development Program.

[6] Chen, D., and Daclin, N. (2007). Barriers driven methodology for enterprise interoperability. In Establishing the foundation of collaborative networks. Springer US.

[7] Daclin, N., Chen, D. and Vallespir, B. (2008). Methodology for Enterprise Interoperability. Proceedings of the 17th World Congress The International Federation of Automatic Control. Seoul, Korea.

[8] Galster, M., Avgeriou, P., \& Tofan, D. (2013). Constraints for the design of variability-intensive service-oriented reference architectures-An industrial case study. Information and Software Technology, 55(2), 428-441. 
[9] Daclin, N. (2005). Contribution to a methodology to develop interoperability of enterprise applications. I-ESA.

[10] Kang, K. C., Lee, J., \& Donohoe, P. (2002). Feature-oriented product line engineering. IEEE software, 19(4), 58-65.

[11] F. Lampathaki, S. Mouzakitis, G. Gionis, Y. Charalabidis, D. Askounis. (2009). Business to business interoperability: a current review of XML data integration standards, Computer Standards \& Interfaces, 31 (6), 1045-1055.

[12] Lapouchnian, A., \& Lespérance, Y. (2006, June). Modeling mental states in agent-oriented requirements engineering. In Advanced Information Systems Engineering (pp. 480-494). Springer Berlin Heidelberg.

[13] Rolland, C., Prakash, N., \& Benjamen, A. (1999). A multi-model view of process modelling. Requirements Engineering, 4(4), 169-187.

[14] Driss, M., Moha, N., Jamoussi, Y., Jézéquel, J. M., \& Ben Ghezala, H. (2010). A requirement-centric approach to web service modeling, discovery, and selection. In Service-Oriented Computing (pp. 258-272). Springer Berlin Heidelberg.

[15] Novakouski M., Lewis G.A, Interoperability in the e-Government Context, SEI, Carnegie Mellon University, Pittsburgh (2012), pp. 1-35

[16] Charalabidis Y., Lampathaki F., Kavalaki A., Askounis D., A review of electronic government interoperability frameworks: patterns and challenges, International Journal of Electronic Governance, 3 (2) (2010), pp. 189-221

[17] Kasmi M., Jamoussi Y., \& Ben Ghezala H. (2016). "Recommendation in Interactive Web Services Composition: A State-of-the-Art Survey." International Conference on Electronic Commerce and Web Technologies. Springer.

[18] Arenas, A. E., Massonet, P., Ponsard, C., \& Aziz, B. (2015). Goal-Oriented Requirement Engineering Support for Business Continuity Planning. InAdvances in Conceptual Modeling (pp. 259-269). Springer International Publishing.

[19] Jureta, I. J., Borgida, A., Ernst, N. A., \& Mylopoulos, J. (2015). The requirements problem for adaptive systems. ACM Transactions on Management Information Systems (TMIS), 5(3), 17.

[20] Hu, H., Ma, Q., Zhang, T., Tan, Y., Xiang, H., Fu, C., \& Feng, Y. (2015). Semantic modelling and automated reasoning of non-functional requirement conflicts in the context of softgoal interdependencies. Software, IET, 9(6), 145-156.

[21] Hasan, M. M. (2015). E-Government Service Research Development: A Literature Review. International Journal of E-Services and Mobile Applications (IJESMA), 7(1), 22-49. 
[22] Souilah M., Boufaida M., (2014). Toward a Better Interoperability of Enterprise Information Systems: A CPNs and Timed CPNs -based Web Service Interoperability Verification in a Choreography, Procedia Technology, Volume 16, Pages 269-278.

[23] Melliti, T., Haddad, S., Suna, A., \& El Fallah-Seghrouchni, A. (2005, September). Web-MASI: Multi-Agent Systems Interoperability Using a Web Services Based Approach. In IAT (Vol. 5, pp. 739-742).

[24] [24] Rezaei, R., Chiew, T. K., \& Lee, S. P. (2014). A review on E-business Interoperability Frameworks. Journal of Systems and Software, 93, 199-216.

[25] Erl, T. (2011). 'SOA Principles of Service Design', Prentice Hall, Englewood Cliffs.-3

[26] Kaura, V., Durga Prasad, C. S., \& Sharma, S. (2015). Service quality, service convenience, price and fairness, customer loyalty, and the mediating role of customer satisfaction. International Journal of Bank Marketing, 33(4), 404-422.

[27] Bleistein, S. J., Cox, K., Verner, J., \& Phalp, K. T. (2006). B-SCP: A requirements analysis framework for validating strategic alignment of organizational IT based on strategy, context, and process. Information and software technology, 48(9), 846-868.

[28] Papadacci-Stephanopoli E., Salinesi C. and Rolland C. (2006) "NENO process: Information systems arbitration process in Enterprise Architecture Project, Information and Communication Technologies, 2006. ICTTA '06. 2nd, Damascus, pp. 298-303.

[29] Saekow, A., \& Boonmee, C. (2009). A pragmatic approach to interoperability practical implementation support (IPIS) for e-Government interoperability. Electronic Journal of E-Government, 7(4), 403-414.

[30] [30] Soares, D., \& Amaral, L. (2011). Information systems interoperability in public administration: identifying the major acting forces through a Delphi study. Journal of theoretical and applied electronic commerce research, 6(1), 61-94.

[31] Ounaies, H. Z., Jamoussi, Y., \& Ben Ghezala, H. (2010). Evaluation framework based on fuzzy measured method in adaptive learning systems. Themes in Science and Technology Education, 1(1), 49-58.

[32] Saekow, A., \& Boonmee, C. (2009). A pragmatic approach to interoperability practical implementation support (IPIS) for e-government interoperability. Electronic Journal of E-government, 7(4), 403-414.

[33] Sanati, F., Lu, J., \& Zeng, X. (2007). A methodological framework for eGovernment service delivery integration. eGovernment Interoperability Campus, Paris. 
[34] Faratin, P., Sierra, C., \& Jennings, N. R. (2002). Using similarity criteria to make issue trade-offs in automated negotiations. Artificial Intelligence, 142(2), 205-237.

[35] Daclin, N., Chen, D., \& Vallespir, B. (2008, July). Methodology for enterprise interoperability. In Proceedings of the 17th World Congress: The International Federation of Automatic Control, Seoul, Korea (pp. 6-11).

[36] Z. Al-Khanjari, N. Al-Hosni, N. Kraiem, Y. Jamoussi. "Developing eGovernment Interoperability Driven Methodology", Journal of Emerging Technologies in Web Intelligence, 2014, 6(3), 318-323.

[37] Benbya, H., \& McKelvey, B. (2006). Using coevolutionary and complexity theories to improve IS alignment: a multi-level approach. Journal of Information technology, 21(4), 284-298.

[38] Wu, L., \& Buyya, R. (2012). Service Level Agreement (SLA) in utility computing systems. IGI Global. Vol.15.

[39] Al-Khanjari, Z., Al-Hosni, N. \& Kraiem, N. (2014). "Developing A Service Oriented e-Government Architecture Towards Achieving EGovernment Interoperability". International Journal of Software Engineering and Its Applications (IJSEIA), 8(5), pp. 29-42, May 2014 